\title{
CONVERGENCE PROPERTIES OF NEIGHBORING SEQUENCES
}

\author{
RALPH R. SABELLA
}

\begin{abstract}
First countable spaces and semimetrizable spaces are examples of topological spaces which can be characterized in terms of convergence properties of sequences "neighboring" a point. In this paper we consider conditions sufficient for metrizability of spaces defined in terms of convergence properties of "neighboring" sequences, in particular, those in which the set of cluster points of one sequence is a subset of that of any "neighboring" sequence. The special case in which the sets of cluster points are equal is shown to be a characterization of metrizability in $T_{0}$-spaces.
\end{abstract}

We use $Z$ to represent the set of natural numbers and $\eta(x)$ the open neighborhoods of $x$. If $\left\{x_{n}\right\}$ is a sequence, $\operatorname{Cp}\left\{x_{n}\right\}$ will denote the set of cluster points of $\left\{x_{n}\right\}$.

Definition 1. If $X$ is a space, $U: X \times Z \rightarrow \bigcup\{\eta(x): x \in X\}$ is an open neighborhood assignment (ONA) on $X$ if $x \in U(x, n) \equiv U_{n}(x)$ for all $x$ and $n$.

If $X$ satisfies property $P$ defined in terms of an ONA $U$, we shall say " $U$ satisfies $P$."

Definition 2. Let $U$ be an ONA on $X$. The sequence $\left\{x_{n}\right\}$ is $U$-linked to the sequence $\left\{y_{n}\right\}$ if $x_{n} \in U_{n}\left(y_{n}\right)$ for all $n$.

DEFINITION 3. An ONA $U$ is coconvergent (contraconvergent) if $\operatorname{Cp}\left\{x_{n}\right\} \subset \operatorname{Cp}\left\{y_{n}\right\}\left(\operatorname{Cp}\left\{x_{n}\right\} \supset \operatorname{Cp}\left\{y_{n}\right\}\right)$ whenever $\left\{y_{n}\right\}$ is $U$-linked to $\left\{x_{n}\right\}$.

Proposition 4. If $U$ is a coconvergent $O N A$ then it is first countable.

Proof. If there is a point $y$ and an $R \in \eta(y)$ such that for each $n$ there is an $x_{n} \in U_{n}(y)-R$ then by coconvergence $y \in \operatorname{Cp}\left\{x_{n}\right\}$. But $x_{n} \notin R$ for any $n$, which is impossible.

The following is an example of a contraconvergent space which is not first countable and hence by Proposition 4, not coconvergent. Let $X=$ $\cup I_{n}$ where $I_{n}=\{(x, y): y=(1 / n) x, 0 \leqq x \leqq 1\}$. Each $I_{n}$ is furnished with the relative topology from $R^{2}$ and $X$ is topologized with the sum topology.

Received by the editors October 16, 1970.

AMS (MOS) subject classifications (1970). Primary 54E35; Secondary 54D99.

Key words and phrases. Open neighborhood assignments, $U$-linked sequences, coconvergent, contraconvergent, Moore Metrization Theorem.

(C) American Mathematical Society 1973 
Define $U$ on $X$ as follows. If $\left(x_{0}, y_{0}\right) \neq(0,0)$ let

$$
U_{k}\left(\left(x_{0}, y_{0}\right)\right)=\left\{(x, y) \in X-(0,0): y=(1 / n) x,\left|x-x_{0}\right|<1 / k\right\} .
$$

For $(0,0)$ we let

$$
U_{k}((0,0))=\{(x, y) \in X: d((0,0),(x, y))<1 / k\} .
$$

The ONA $U$ is contraconvergent but $(0,0)$ has no countable local base.

Proposition 5. Let $X$ be a set and $d$ a real valued nonnegative function defined on $X \times X$ such that (a) $d(x, x)=0$ for all $x$, and (b) $d(x, y) \leqq d(x, z)+$ $d(z, y)$ for all $x, y, z$. Then the family $\left\{U_{n}(x): x \in X, n \in Z\right\}$ where $U_{n}(x)=$ $\{y: d(x, y)<1 / n\}$ is a base for a topology and the associated $O N A$ is coconvergent.

The proof that $U$ generates a topology and is coconvergent follows standard metric arguments.

COROLlaRY 6. Every pseudometric and every quasi-metric space is coconvergent.

M. Sion and G. Zelmer [5] proved that a $T_{1}$ space with a $\sigma$-point finite base is a quasi-metric space.

COROLlaRY 7. Every $T_{1}$ space with a $\sigma$-point finite base is coconvergent.

The "Sorgenfrey line," i.e., the points of the real line with the topology generated by all half-open intervals, open on the right is quasi-metrizable and hence coconvergent.

An ONA $U^{\prime}$ will be called a refinement of an ONA $U$ if $U_{n}^{\prime}(x) \subset U_{n}(x)$ for all $x$ and $n$. We shall further say $U$ is nested if $U_{n+1}(x) \subset U_{n}(x)$ for all $x$ and $n$.

Proposition 8. (a) If a space $X$ has an $O N A$, every refinement of which satisfies property $P$, then $X$ has a nested $O N A$ satisfying $P$.

(b) If a space $X$ has nested $O N A$ 's satisfying properties $P$ and $Q$ respectively, then $x$ has a nested $O N A$ that satisfies both $P$ and $Q$.

(c) A space with a coconvergent (contraconvergent) ONA has a nested coconvergent (contraconvergent) ONA.

Proof. (a) Define $U^{\prime}$ by $U_{n}^{\prime}(x)=\bigcap\left\{U_{k}(x): k=1, \cdots, n\right\}$ where $U$ is the given ONA.

(b) Let $U$ and $V$ satisfy $P$ and $Q$ respectively;

satisfies both $P$ and $Q$.

$$
W_{n}(x)=\left|\bigcap_{k=1}^{n} U_{k}(x)\right| \cap\left|\bigcap_{k=1}^{n} V_{k}(x)\right|
$$


(c) Let $U$ be coconvergent, $U^{\prime}$ be a refinement of $U$ and let $\left\{y_{n}\right\}$ be $U^{\prime}$-linked to $\left\{x_{n}\right\}$. Then $\left\{y_{n}\right\}$ is $U$-linked to $\left\{x_{n}\right\}$ and $\operatorname{Cp}\left\{y_{n}\right\} \subset \operatorname{Cp}\left\{x_{n}\right\}$, implying $U^{\prime}$ is coconvergent. The proof is the same for contraconvergence.

The following is a restatement of the Moore Metrization Theorem. (See Jones [4].)

THEOREM. A space $X$ is metrizable iff it is $T_{2}$ and there exists a sequence $\left\{\mathscr{K}_{n}\right\}$ such that (a) each $\mathscr{K}_{n}$ is an open covering of $X$, and (b) for any point $p$ and $R \in \eta(p)$ there exists an $n$ such that if $G, H \in \mathscr{K}_{n}, p \in G$ and $G \cap H \neq \varnothing$ then $H \subset R$.

Proposition 9. A space $X$ is metrizable if it is a coconvergent, contraconvergent $T_{0}$ space.

Proof. If $X$ is a metric space with distance function $d$, define $U$ by letting $U_{n}(x)=\{y: d(x, y)<1 / n\}$ for all $x$ and $n$. It easily follows that $U$ is coconvergent and contraconvergent.

Conversely, by Proposition 8 there is an ONA $U$ which is coconvergent and contraconvergent. Also, $X$ is $T_{2}$, for if there are distinct $x$ and $y$ such that for all $n$ there is a $z_{n} \in U_{n}(x) \cap U_{n}(y)$, it would follow that $\operatorname{Cp}\{x\}=$ $\mathrm{Cp}\left\{z_{n}\right\}=\operatorname{Cp}\{y\}$ which is impossible.

Letting $\mathscr{K}_{n}=\left\{U_{n}(x): x \in X\right\},\left\{\mathscr{K}_{n}\right\}$ satisfies (a) of the Moore Metrization Theorem. Now, suppose $R \in \eta(p)$ and there are sequences $\left\{x_{n}\right\},\left\{y_{n}\right\}$ and $\left\{z_{n}\right\}$ such that $p \in U_{n}\left(x_{n}\right), z_{n} \in U_{n}\left(x_{n}\right) \cap U_{n}\left(y_{n}\right)$ and $U_{n}\left(y_{n}\right) \cap(X-R) \neq \varnothing$ for all $n$. Let $w_{n} \in U_{n}\left(y_{n}\right)-R$. It follows by coconvergence and contraconvergence that $p=\mathrm{Cp}\left\{w_{n}\right\} \in X-R$ which is a contradiction.

Proposition 10. Let $X$ be a countably compact, $T_{2}$ coconvergent space. Then $X$ is metrizable.

Proof. By Proposition $4, X$ is first countable. From countable compactness, first countability and $T_{2}, X$ is regular.

Let $U$ be a coconvergent ONA and define $\left\{\mathscr{K}_{n}\right\}$ as in Proposition 9. For any point $p$ and $R \in \eta(p)$ there is an $S \in \eta(p)$ such that $p \in S \subset \bar{S} \subset R$ and an $N \in Z$ such that $U_{N}(y) \subset S$ for any $y$ for which $p \in U_{N}(y)$. Otherwise there are sequences $\left\{x_{n}\right\}$ and $\left\{y_{n}\right\}$ such that for all $n, x_{n} \in U_{n}\left(y_{n}\right)-S$ and $\{p\}$ is $U$-linked to $\left\{y_{n}\right\}$. By coconvergence and countable compactness it follows that $p \in \operatorname{Cp}\left\{x_{n}\right\}$ contradicting $p \in S$.

If $\left\{\mathscr{K}_{n}\right\}$ does not satisfy the Moore Metrization Theorem, there exists a point $p$ and an $R \in \eta(p)$ such that for each $n$ there are $U_{n}\left(x_{n}\right)$ and $U_{n}\left(y_{n}\right)$ for which $p \in U_{n}\left(x_{n}\right), U_{n}\left(x_{n}\right) \cap U_{n}\left(y_{n}\right) \neq \varnothing$ and $U_{n}\left(y_{n}\right)$ is not contained in $R$. Choosing $S \in \eta(p)$ such that $p \in S \subset S \subset R$, it follows by the previous paragraph, countable compactness and coconvergence that there is a $y \in \mathrm{Cp}\left\{y_{n}\right\}$ such that $y \in \bar{S} \cap(X-R)$. But this contradicts $\overline{S \subset R}$ which completes the proof of the proposition. 
The standard example of a $T_{1}$, non- $T_{2}$ space made up of the unit interval with two left "end points" provides an example of a coconvergent, countably compact, $T_{1}$, nonmetrizable space. The topology of this example is quasi-metrizable as was the topology of the example given after Corollary 7. It is not known if quasi-metrizability and coconvergence are in fact equivalent.

We use the notation $\Delta\left(\left\{x_{n}\right\}, x_{0}\right) \equiv\left\{x_{k}: k=0,1, \cdots\right\}$ whenever $\left\{x_{n}\right\}$ converges to $x_{0}$.

Lemma 11. Let $U$ be an $O N A$ on $X$ then the following are equivalent: (a) The ONA $U$ is coconvergent. (b) For any countably compact $K$ and open $R$ containing $K, \bigcup\left\{U_{n}(x): x \in K\right\} \subset R$ for some $n \in Z$. (c) For any $\Delta\left(\left\{x_{n}\right\}, x_{0}\right)$ and open $R$ containing $\Delta\left(\left\{x_{n}\right\}, x_{0}\right)$,

$$
\bigcup\left\{U_{n}(x): x \in \Delta\left(\left\{x_{n}\right\}, x_{0}\right)\right\} \subset R \text { for some } n \in Z .
$$

Proof. Statement (a) implies (b). Assume $K$ is countably compact and $R$ is open and contains $K$. If there exist sequences $\left\{x_{n}\right\}$ and $\left\{y_{n}\right\}$ such that $x_{n} \in U_{n}\left(y_{n}\right)-R$ and $y_{n} \in K$ for all $n$, then $\left\{y_{n}\right\}$ clusters at some $y \in K$. By coconvergence $y \in \mathrm{Cp}\left\{x_{n}\right\}$ which is impossible. Statement (b) trivially implies (c).

Finally, if $U$ is an ONA satisfying the condition of (c), by Proposition 8 we can take $U$ to be nested. Also, $X$ is obviously first countable. If $x \in$ $\operatorname{Cp}\left\{x_{n}\right\}$ we consider a subsequence $\left\{x_{n_{k}}\right\}$ of $\left\{x_{n}\right\}$ which converges to $x_{0}$. If $R \in \eta\left(x_{0}\right)$ then $\Delta\left(\left\{x_{n_{k}}\right\}_{k=M}^{\infty}, x_{0}\right) \subset R$ for $M$ large enough. Hence for some $r \in Z$,

$$
\bigcup\left\{U_{r}(x): x \in \Delta\left(\left\{x_{n_{k}}\right\}_{k=M}^{\infty}, x_{0}\right)\right\} \subset R .
$$

Picking $k$ such that $n_{k}>r, U_{n_{k}}\left(x_{n_{k}}\right) \subset R$. For any sequence $\left\{y_{n}\right\} U$-linked to $\left\{x_{n}\right\}$ it now follows that $x_{0} \in \mathrm{Cp}\left\{y_{n}\right\}$.

Lemma 12. A space $X$ is metrizable iff $X$ is $T_{0}$ and there is an ONA $U$ on $X$ such that if $R$ is open and contains $\Delta\left(\left\{x_{n}\right\}, x_{0}\right)$ then for some $k \in Z$, $U_{k}(z) \subset R$ for all $z \in X$ such that $U_{k}(z) \cap \Delta\left(\left\{x_{n}\right\}, x_{0}\right) \neq \varnothing$.

Proof. If $X$ is metrizable the ONA induced by the metric satisfies the condition of the hypothesis.

Conversely, if $U$ satisfies the condition it is clear that every refinement of $U$ satisfies the condition. Therefore we may take $U$ to be nested. The condition implies for any open set $R$ containing $\Delta\left(\left\{x_{k}\right\}, x_{0}\right)$, there is an $n$ such that $\bigcup\left\{U_{n}(z): z \in \Delta\left(\left\{x_{k}\right\}, x_{0}\right)\right\} \subset R$. By Lemma 11, $U$ is coconvergent.

If $\left\{y_{n}\right\}$ is $U$-linked to $\left\{x_{n}\right\}$ and $y \in \operatorname{Cp}\left\{y_{n}\right\}$, we consider a subsequence $\left\{y_{n_{k}}\right\}$ of $\left\{y_{n}\right\}$ converging to $y$. It now follows from the given condition on $U$ that if $R \in \eta(y), U_{n_{k}}\left(x_{n_{k}}\right) \subset R$ for $n_{k}$ large enough. Hence, $U$ is contraconvergent and by Proposition 9, $X$ is metrizable. 
Proposition 13. The space $X$ is metrizable if it is $T_{2}$, coconvergent and has a locally finite open cover $\mathscr{F}$ such that $\bar{F}$ is compact for every $F \in \mathscr{F}$.

Proof. For each $x \in X$ let $W(x) \in \eta(x)$ intersecting only finitely many elements of $\mathscr{F}$ and let $F(x) \in \eta(x) \cap \mathscr{F}$.

If $S$ is a nested coconvergent ONA, let $U_{n}(x)=F(x) \cap W(x) \cap S_{n}(x)$ for $n \in Z$. The ONA $U$ is a refinement of $S$ and is coconvergent.

Let $R$ be open and contain $\Delta\left(\left\{x_{n}\right\}, x_{0}\right)$. By Lemma 11 there is an $m$ such that

$$
\bigcup\left\{U_{m}(x): x \in \Delta\left(\left\{x_{n}\right\}, x_{0}\right)\right\} \subset R .
$$

Since $U_{m}\left(x_{0}\right)$ contains almost all the $x_{k}$ 's, there is an $r \in Z$ such that

$$
\Delta\left(\left\{x_{n}\right\}, x_{0}\right) \subset \bigcup\left\{U_{m}\left(x_{k}\right): k=0,1, \cdots, r\right\} .
$$

For each $j \in\{0,1, \cdots, r\}$ there is a finite subcollection $\mathscr{F}_{j}$ of $\mathscr{F}$ such that if $F \in \mathscr{F}-\mathscr{F}_{j}$ then $F \cap W\left(x_{j}\right)=\varnothing$. Hence, $\mathscr{E}=\bigcup\left\{\mathscr{F}_{j}: j=0,1, \cdots, r\right\}$ is a finite family of sets.

If there exist $\left\{z_{n}\right\}$ and $\left\{y_{n}\right\}$ such that, for each $n, z_{n} \in U_{n}\left(y_{n}\right)-R$ and $U_{n}\left(y_{n}\right) \cap \Delta\left(\left\{x_{n}\right\}, x_{0}\right) \neq \varnothing$, it would follow from $U_{n}\left(y_{n}\right) \subset F\left(y_{n}\right)$ and $U_{m}\left(x_{k}\right) \subset W\left(x_{k}\right)$ that $F\left(y_{n}\right) \in \mathscr{F}_{k} \subset \mathscr{E}$ for some $k \in\{0,1, \cdots, r\}$. Hence, $\left\{F\left(y_{n}\right): n \in Z\right\} \subset \mathscr{E}$ and there must be a subsequence $\left\{y_{n_{k}}\right\}$ of $\left\{y_{n}\right\}$ such that $F\left(y_{n_{1}}\right)=F\left(y_{n_{2}}\right)=\cdots$. But $\left\{y_{n_{k}}\right\}$ is then a sequence in the compact set $\mathrm{Cl}\left(F\left(y_{n_{1}}\right)\right)$ and must cluster at some $y \in \mathrm{Cp}\left\{y_{n}\right\}$.

For each $n$ there is an $a_{n} \in \Delta\left(\left\{x_{n}\right\}, x_{0}\right) \cap U_{n}\left(y_{n}\right)$. By coconvergence $y \in \mathrm{Cp}\left\{a_{n}\right\}$ and hence $y \in R$.

Finally, there is a subsequence $\left\{y_{n_{k}}\right\}$ of $\left\{y_{n}\right\}$ converging to $y$. By Lemma $11, U_{k}\left(y_{k}\right) \subset R$ for some $k$ contradicting $z_{k} \in U_{k}\left(y_{k}\right)-R$. Thus $U$ satisfies the condition of Lemma 12.

Corollary 14. Let $X$ be locally compact. Then, $X$ is metrizable iff $X$ is paracompact and coconvergent.

As illustrated by the space in the example following Corollary 7 , which is paracompact and coconvergent but not metrizable, the local compactness condition of this last corollary cannot be dropped.

\section{REFERENCES}

1. C. E. Aull, Closed set countability axioms, Nederl. Akad. Wetensch. Proc. Ser. A 69=Indag. Math. 28 (1966), 311-316. MR 33 \#7973.

2. J. Dugundji, Topology, Allyn and Bacon, Boston, Mass., 1966. MR 33 \#1824.

3. R. W. Heath, Arc-wise connectedness in semi-metric spaces, Pacific J. Math. 12 (1962), 1301-1319. MR 29 \#4032.

4. F. B. Jones, Metrization, Amer. Math. Monthly 73 (1966), 571-576. MR 33 \#7980.

5. M. Sion and G. Zelmer, On quasi-metrizability, Canad. J. Math. 19 (1967), 12431249. MR 36 \#4522.

Department of Mathematics, California State University, Northridge, CALIFORNIA 91324 